Research Letter

\title{
Promotion and inhibition of mutation in pathogens
}

\author{
Maurice Samuel Devaraj
}

Received 26 August 2013, Accepted 1 October 2013

(C) 2013, Devaraj M.S.

C 2013, Russian Open Medical Journal

\begin{abstract}
This paper presents the research findings of a sequence of three experiments that were used to assess the impact of a cell's stage in its life cycle on mutatability and its possible mechanism. The first experiment consisted of a modified fluctuation test (Luria-Delbruck) to test for concurrency of jackpot streptomycin-resistant mutant colony in synchronously treated cultures. The second experiment consisted of performing the same test with each set of parallel cultures consisting of different media that included adenosine tri-phosphate (ATP), adenosine di-phosphate (ADP), adenosine mono phosphate (AMP), and adenosine, to see if these compounds, whose levels were modified in bacterial cells during cell division, promoted or suppressed mutation. A third experiment was performed using the same methodology to test the effect of magnesium on the occurrence of the mutant colonies. In the first experiment, the occurrence of jackpot counts of streptomycin-resistant bacteria was concurrent among parallel cultures, indicating that mutation was directed. In the second experiment, ATP and adenosine suppressed mutation, though in different levels. ADP and AMP promoted mutation in different levels. In the third experiment, addition of extracellular magnesium (in the form of magnesium sulphate) promoted mutation, even in the presence of adenosine, which was identified as a mutation suppressor in experiment 2 . We conclude that bacterial mutation is a directed response to external stress and that mutatability is dependent on the cell's phase during the time of external stress. We also conclude that mutation is possible during the cell division process due to the availability of cellular magnesium for ribosomal ribonucleic acid (rRNA) modification. Findings from this research may be used to prevent development of drug resistance, whether epigenetic or arising due to deoxyribonucleic acid (DNA) modification, in several pathogens, especially Mycobacterium tuberculosis through the co-administration of adenosine along with antibiotic treatment.
\end{abstract}

Keywords: directed mutation, Escherichia Coli, drug-resistance, streptomycin, magnesium ion

Cite as Devaraj MS. Promotion and inhibition of mutation in pathogens. Russian Open Medical Journal 2014; 3: 0102.

Correspondence to Maurice Samuel Devaraj. Address: 5/3 Shenbagah Poovu Street, Bhagavathi Nagar, East Tambaram, Chennai 600059, India. Phone (mobile): +91 (0) 9841119309 . E-mail: melanon@gmail.com

\section{Introduction}

Direct mutagenesis or directed mutation is the hypothesis that mutations arise as a specific response to external stresses. Random mutagenesis is the hypothesis that mutations occur randomly, and are pre-existing before being selected for fitness. The acquisition of drug resistance by bacteria has been considered to be a result of random mutation. While drug resistance has been quantitatively researched for different taxa against different antibiotics, there is little or no research on the mechanism by which bacteria acquire drug resistance.

Classical experiments [1-4] have undertaken to prove that mutation is random and not directed. These experiments have employed statistical methods and have involved certain assumptions in the interpretation of the results. The findings have been corroborated by recent experiments [5-16] have attempted to demonstrate the possibility of directed mutation using beneficial interactions, which have been disproved and shown to be mechanistically Darwinian, or as being a product of mechanisms such as hypermutatability. In some instances these 'directed mutations' have been identified as being truly random mutation [5, 17-19]. Other mechanisms that have been proposed to apparent directed mutation include epigenetic modifications.

The starting point for the investigation was taken as Luria and Delbruck's fluctuating test. Our initial hypothesis was that the jackpot number of mutant colonies presented as statistical evidence for random mutation in the fluctuating test actually demonstrated a specific stage in the cell's life cycle present across the culture, during which the cells were able to mutate in response to the stress. In those cultures demonstrating non-jackpot number of colonies, the majority of cells had passed or was hypothesized as yet to pass the specific stage in their life cycle. If the theory was correct, a set of parallel cultures grown from a small innocula and treated at fixed time intervals with streptomycin should have exhibited jackpot streptomycin-resistant colonies at periodic time intervals coinciding with the specific stage of the cell that was occurring synchronously across a majority of the cultures. However, as bacterial cell division is not symmetrical [20], the experimental results differed from the expected results (data not shown). To account for asymmetrical cell division, the test was modified to check for concurrent occurrence and non-occurrence of jackpot number of mutant colonies in synchronously plated cultures. If the random mutation hypothesis was correct, then there would be no concurrency in parallel plates. If the mutation was dependent on the cell being exposed to streptomycin at a specific stage in the cell cycle, the occurrence of mutant jackpot number of streptomycin colonies would be concurrent across synchronous parallel cultures. 
Table 1. Colony count of streptomycin resistant bacteria with each row containing the colony counts for a set of plates synchronously treated with streptomycin

\begin{tabular}{ccccc}
\hline Number & Set 1 & Set 2 & Set 3 & Set 4 \\
\hline 1 & $400+$ & $400+$ & $400+$ & $400+$ \\
2 & 48 & $400+$ & 98 & 27 \\
3 & 15 & 35 & 11 & 4 \\
4 & 65 & 3 & 18 & 4 \\
5 & 3 & 9 & 18 & 14 \\
6 & 2 & 1 & 0 & 1 \\
7 & 16 & 4 & 12 & 18 \\
8 & 24 & 25 & NR* & 5 \\
9 & 23 & 3 & 1 & 19 \\
10 & 9 & 17 & 10 & 5 \\
\hline$*$
\end{tabular}

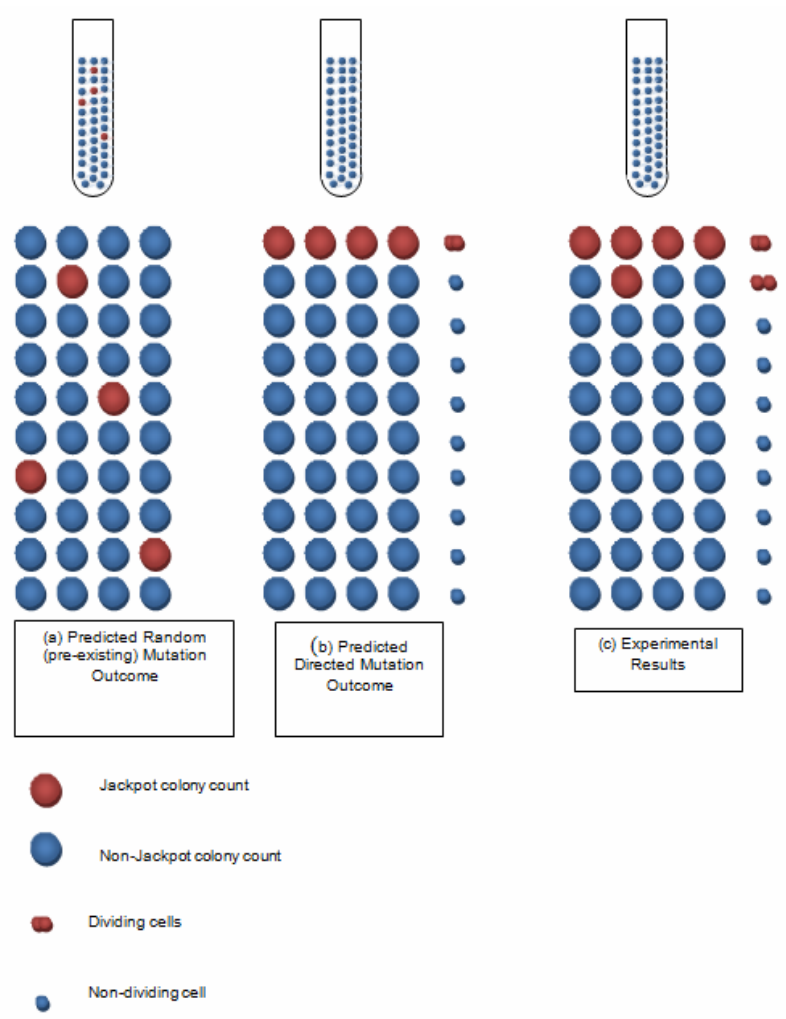

Figure 1. Illustration depicting the predicted outcomes for Experiment 1 if mutation is random (a), mutation is directed (b), and the actual experimental results (c).

Streptomycin is an aminoglycoside that inhibits protein synthesis and causes cell death in bacteria by binding to the $16 \mathrm{~S}$ ribosomal ribonucleic acid (rRNA) of the $30 \mathrm{~S}$ subunit of bacterial ribosome. Streptomycin acts by frame shift mutation during translation and defective protein synthesis which ends in cell death. It binds to the 30S ribosomal subunit, thereby preventing $\mathrm{N}$-formylmethionine (fMet) transfer ribonucleic acid (tRNA) from binding with the $30 \mathrm{~S}$ subunit when initiating protein synthesis [21].

Here, we demonstrate that occurrence of streptomycin resistant mutant strains in Escherichia coli occurs during a specific point in the cell's life cycle. We also demonstrate that the occurrence of streptomycin resistance can be increased and decreased by inclusion of adenosine mono phosphate (AMP) and adenosine (ADO) respectively. We also demonstrate that the mechanism of action by these compounds is by increasing or decreasing the amount of cellular magnesium $\left(\mathrm{Mg}^{2+}\right)$. This increase or decrease in mutatability was found to be unrelated to the number of mutations arising from exposure to streptomycin only. These findings imply that mutation in general and drug resistance in $E$. coli in particular may be directed by external stress.

\section{Material and Methods}

\section{Experiment 1}

Wild type E. coli was isolated from fecal matter. The isolated E.coli was cultured in nutrient beef broth for 24 hours. The bulk culture was used to innoculate forty slants containing streptomycin sulphate solution. Sets of four slants were inoculated with $10 \mu \mathrm{l}$ of innocula for each slant, synchronously* and each set was inoculated after a five minute interval. A total of 10 sets (40 cultures) were inoculated. After an incubation period of 8 hours, $10 \mu \mathrm{l}$ of innocula from each slant was cultured on individual nutrient agar plates using swabs. The plates were examined after 36 hours and 48 hours and colony counts were taken.

\section{Experiment 2}

A small innocula of wild type $E$. coli that was isolated from fecal matter and grown in a nutrient beef broth culture for 24 hours. The bulk culture was used to inoculate five types of media kept in slants. Two sets of each type of media were inoculated.

(1) Streptomycin (Sm),

(2) Streptomycin with adenosine tri-phosphate (ATP),

(3) Streptomycin with adenosine di-phosphate (ADP),

(4) Streptomycin with adenosine mono-phosphate (AMP),

(5) Streptomycin with adenosine (ADO).

Each set of ten media slants was inoculated with $10 \mathrm{ul}$ of innocula for each slant in parallel (identical time of innoculation)*. The cultures were allowed to stand for 7-8 hours before $10 \mu \mathrm{l}$ of innocula from each slant was plated on individual nutrient agar plates.

A total of fifteen sets of the cultures (total of 150 cultures) were plated. The plates were examined after 24 hours and a colony count was taken.

\section{Experiment 3}

Experiment 2 was repeated with modification in the media used for the slants. The following media were used:

(1) Streptomycin,

(2) Streptomycin with adenosine,

(3) Streptomycin with adenosine and magnesium (in the form of magnesium sulphate),

(4) Streptomycin with magnesium,

(5) Adenosine.

*The experiments are extremely time-sensitive and may produce varying results if there are deviations (time lag between plates when inoculating a set). Change to time intervals but with adherence time sensitiveness should produce accurate data but with increase or decrease in detail for decreased and increased time intervals respectively. 
Table 2. Colony count of streptomycin resistant bacteria tested for mutation rates in different media, with each row containing the colony counts for a set of plates synchronously treated with streptomycin

\begin{tabular}{|c|c|c|c|c|c|c|c|c|c|c|}
\hline \multirow[t]{2}{*}{$\begin{array}{l}\text { Media composition } \\
\text { Set Label }\end{array}$} & \multicolumn{2}{|c|}{$\begin{array}{l}\text { Streptomycin only } \\
(2 \mathrm{mg} / 1000 \mathrm{ml})\end{array}$} & \multicolumn{2}{|c|}{$\begin{array}{c}\text { ATP } \\
\text { (1mg/100ml of Sm } \\
\text { solution with conc. of } \\
2 \mathrm{mg} / 1000 \mathrm{ml})\end{array}$} & \multicolumn{2}{|c|}{$\begin{array}{c}A D P \\
(1 \mathrm{mg} / 100 \mathrm{ml} \text { of } \mathrm{Sm} \\
\text { solution with conc. of } \\
2 \mathrm{mg} / 1000 \mathrm{ml})\end{array}$} & \multicolumn{2}{|c|}{$\begin{array}{c}A M P \\
\text { (1mg/100ml of } \mathrm{Sm} \\
\text { solution with conc. of } \\
2 \mathrm{mg} / 1000 \mathrm{ml})\end{array}$} & \multicolumn{2}{|c|}{$\begin{array}{c}A D O \\
(1 \mathrm{mg} / 100 \mathrm{ml} \text { of } \mathrm{Sm} \\
\text { solution with conc. of } \\
2 \mathrm{mg} / 1000 \mathrm{ml})\end{array}$} \\
\hline & 1 & 2 & 3 & 4 & 5 & 6 & 7 & 8 & 9 & 10 \\
\hline A & 127 & 286 & 19 & 15 & 17 & 4 & 92 & 62 & 6 & 6 \\
\hline B & 2 & 1 & 0 & 8 & 1 & 5 & 4 & 4 & 0 & 0 \\
\hline C & 0 & 0 & 0 & 0 & 3 & 48 & 58 & 4 & 3 & 2 \\
\hline D & 2 & 2 & 2 & 0 & 14 & 2 & 64 & 54 & 0 & 1 \\
\hline E & 1 & 1 & 0 & 2 & 1 & 1 & 2 & 8 & 1 & 2 \\
\hline $\mathrm{F}$ & 0 & 1 & 0 & 0 & 0 & 2 & Con & 1 & 2 & 0 \\
\hline G & 2 & 1 & 0 & 3 & 1 & 1 & 65 & 45 & 13 & 2 \\
\hline $\mathrm{H}$ & 0 & 0 & 1 & 0 & 1 & 26 & 63 & 63 & 1 & 0 \\
\hline I & 2 & 0 & 10 & 6 & 54 & 39 & 51 & 36 & 0 & 1 \\
\hline J & 0 & 1 & 2 & 1 & 22 & 1 & 41 & 37 & 1 & 0 \\
\hline K & 1 & 1 & 6 & 0 & 2 & 41 & 148 & 93 & 6 & 0 \\
\hline $\mathrm{L}$ & 1 & 0 & Con & 6 & 6 & 27 & 67 & 176 & 0 & 0 \\
\hline$M$ & 1 & 1 & 3 & 3 & 2 & 29 & 121 & 183 & 0 & 4 \\
\hline $\mathrm{N}$ & 0 & 1 & 0 & 23 & 46 & 0 & 32 & 123 & 0 & 0 \\
\hline 0 & 8 & 5 & 4 & 1 & 138 & 19 & 92 & 28 & 0 & 6 \\
\hline Avg. mean for series & 9.8 & 20.1 & 29.8 & 4.5 & 20.5 & 16.3 & 86.7 & 61.1 & 2.2 & 1.6 \\
\hline Avg. mean for media & \multicolumn{2}{|c|}{14.9} & \multicolumn{2}{|c|}{17.2} & \multicolumn{2}{|c|}{18.4} & \multicolumn{2}{|c|}{73.9} & \multicolumn{2}{|c|}{1.9} \\
\hline
\end{tabular}

All slants consisted of $0.5 \mathrm{ml}$ of media.

\section{Results}

In Experiment 1 results shown in Table 1. The cultures with jackpot number of mutant colonies were found to occur concurrently across a set of synchronously treated plates, which was in agreement with the hypothesis for directed mutation as shown in Figure 1.

In Experiment 2 results shown in Table 2. Cultures treated with streptomycin+ATP, and streptomycin + ADP were statistically found to have slightly higher number of mutant colonies. Cultures treated with streptomycin+AMP had significantly higher percentage of mutant colonies $(500 \%$ increase compared to cultures treated with only streptomycin). Cultures treated with streptomycin+adenosine were found to have a considerably lesser number of mutant colonies ( $785 \%$ decrease compared to cultures treated with only streptomycin).

In Experiment 3 (data not shown), the cultures, in which magnesium and streptomycin, showed jackpot number of colonies (non-confluent), while the cultures treated with streptomycin only and streptomycin with adenosine displayed high level of mutation suppression. The cultures with only adenosine showed normal confluent growth.

\section{Discussion}

Cultures treated with ATP appear to have an effect of suppressing mutation, though statistically, they showed a slightly higher level of mutation. Addition of extracellular ATP that is not bound to $\mathrm{Mg}^{2+}$ probably decreases the level of free $\mathrm{Mg}^{2+}$ (which would be otherwise available on the hydrolysis of $\mathrm{MgATP}^{2-}$ available in the cell [22], due to ATP being hydrolysed instead of MgATP $^{2-}$. However the occurrence of a confluent growth (Table 2: L3), and higher number of mutant colonies (Table 2: A3, A4, 13, N4) may be due to cellular MgATP ${ }^{2-}$ being hydrolysed (due to cell division taking place) prior to the addition of extracellular ATP, which results in free $\mathrm{Mg}^{2+}$ being available and thereby results in a high number of mutant cells.
Cultures treated with ADP have higher instances of mutant colonies (13 out of 30 cultures) indicating that the presence of ADP may generally increase mutation to streptomycin. This may be caused by the cell's response of increasing MgATP ${ }^{2-}$ synthesis in response to MgATP ${ }^{2-} / A D P$ imbalance caused by the addition of extracellular ADP. In the case of AMP, the cell's capability to mutate increased significantly. Addition of extracellular AMP may have increased the level of $\mathrm{Mg}^{2+}$ due to increase in the level of MgATP $^{2-}$ and its subsequent hydrolysis caused by the added AMP [23].

Adenosine significantly reduced the cell's capability to mutate. Compared to ATP, which sometimes displayed inability to suppress mutation (Table 2: A3, A4, 13, N4), adenosine consistently displayed an ability to suppress mutation. The ability of extracellular magnesium to promote mutation, even in the presence of adenosine as seen in Experiment 3 provides the possible mechanism of suppression by adenosine. When $\mathrm{Mg}^{2+}$ is available in excess, the ability of adenosine to supress mutation is negated. This indicates that adenosine supressed mutation by reducing the amount of free cellular $\mathrm{Mg}^{2+}$. This may be effected by adenosine binding to the free cellular $\mathrm{Mg}^{2+}$ resulting in decrease of free cellular $\mathrm{Mg}^{2+}$. The effect of $\mathrm{Mg}^{2+}$ may be to cause structural modification of the $30 \mathrm{~S}$ ribosome of streptomycin sensitive cells to enable fMet tRNA bind to the $30 \mathrm{~S}$ subunit and initiate protein synthesis [24] or by increased polymerase activity that enables a mutational response to the streptomycin action.

The results demonstrated in the classical experiments demonstrated a statistical distribution consistent with random mutation, as two possibilities were not considered. The first possibility was that mutation occurred at a specific stage in the cell's life cycle, and the second possibility was that bacterial cell division was asynchronous. Asynchronous cell division and our finding that mutation possibly occurs only during the cell division process results in a pattern of mutation that appears random when tested at specific points. Asynchronous cell division may result in occasional synchronous cell divisions across a culture, 
which showed up as random jackpot number of mutant cells in the classic fluctuation test [11]. The classical re-spreading test's [12] hypothesis did not account for mutatability at a specific stage in the cell cycle. The hypothesis behind the experiment, and therefore the experiment itself becomes invalid.

Modern experiments against directed mutation have been very specific counters to prior experiments that have argued for directed mutation. However, the same omissions in considering mutatability with respect to cell life cycle, and asynchronous mutation have been responsible for the failure for experiments in supporting directed mutations, and the false-positive success of the research supporting random mutation.

The possible mechanism of $\mathrm{Mg}^{2+}$ through increased polymerase activity as a response to streptomycin action may be illustrated by the use of a tape recorder analogy. If DNA can be considered as a tape in a tape recorder, ribosomes and polymerases as the read-write head and cellular ions, especially magnesium as the power source or input signals, external inputs may result in increase or decrease in the level of cellular magnesium through cellular signalling. The level of cellular magnesium in turn may be responsible for the fidelity of both transcription and translation processes, since magnesium is involved in ribosome function, catalysis of enzyme action and deoxyribonucleic acid (DNA) repair and synthesis [24]. The level of magnesium may therefore increase or decrease the level of fidelity during transcription, which may result in mutation, or during translation, which may result in epigenetic modifications. The presence (crest) or absence (trough) of magnesium would be similar to the wave of an electronic signal that 'writes' the data on a magnetic tape, which when read, faithfully reproduces the data (or audio). Since normal levels of magnesium ensures fidelity in the process, absence or lack of magnesium may result in lack of fidelity and thereby mutation such as through the mechanism of DNA methylation $[25,26]$, while higher levels of magnesium may result in mutation through change in rate of polymerase activity as evidenced by PCR optimization tests.

\section{Conclusion}

This research demonstrates that mutation to an external stress such as streptomycin can be manipulated using $\mathrm{Mg}^{2+}$ to increase mutatability, or by using adenosine to reduce mutatability. $\mathrm{Mg}^{2+}$ is possibly used by the cell to modify or repair rRNA during transcription as a response to damage or cell signalling. The ability to manipulate mutatability indicates that mutation is not random and is directed. Bacterial mutation that confers drug-resistance arises when the drug is encountered by the cell during the cell division process. Therefore the measurement of drug-resistant in bacteria from a source cannot be performed using random samples, but has to be performed as a series of timed experiments, where the level of drug-resistance has to be consistent across the cell's life cycle for the bacteria to be truly drug-resistant.

Regardless of whether the mutation that is promoted (by $\mathrm{Mg}^{2+}$ ) or supressed (by adenosine) is epigenetic or due to DNA modification (mutation), the ability of adenosine to reduce mutatability may be researched further with other pathogens for suppression of antibiotic resistance. If adenosine is found to be effective across different pathogens, it can be co-administered or may be made an additional ingredient, where it is mechanically bonded to the compound. Adenosine is being manufactured and sold commercially for the treatment of supraventricular tachycardia, and much is known about its contraindications [27]. Adenosine may be used along with an agonist during antibiotic administration to prevent drug-resistance. Alternatively, other methods of reducing magnesium levels during drug administration may also be examined.

Conflict of interest: none declared.

\section{References}

1. Shapiro JA. Observations on the formation of clones containing araBlacZ cistron fusions. Mol Gen Genet 1984; 194(1-2): 79-90. (PMID: 6233472)

2. Cairns J, Overbaugh J, Miller S. The origin of mutants. Nature 1988; 335: 142-145. (doi: 10.1038/335142a0)

3. Dijkmans R, Kreps S, Mergeay M. Poison-like fluctuation patterns of revertants of leucine auxotrophy (leu-500) in Salmonella typhimurium caused by delay in mutant cell division. Genetics 1994; 137(2): 353 359. (PMID: 8070649) (PMCID: PMC1205961)

4. Lenski RE, Slatkin $M$, Ayala FJ. Another alternative to directed mutation. Nature 1989; 337: 123-124. (doi: 10.1038/337123b0)

5. Hall BG. On alternatives to selection-induced mutation in the $\mathrm{Bg}$ operon of Escherichia coli. Mol Biol Evol 1994; 11(2): 159-168. (PMID: 8170359)

6. Lederberg J. Replica plating and indirect selection of bacterial mutants: isolation of preadaptive mutants in bacteria by sib selection. Genetics 1989; 121(3): 395-399. (PMCID: PMC1203627) (PMID: 2653959)

7. Lenski RE. Are some mutations directed? Trends Ecol Evol 1989; 4(5): 148-150. (PMID: 21227341) (doi: 10.1016/0169-5347(89)90218-8)

8. Lenski RE, Mittler JE. The directed mutation controversy and NeoDarwinism. Science 1993; 259: 188-194. (PMID: 7678468) (doi: 10.1126/science.7678468)

9. Lederberg J, Lederberg EM. Replica plating and indirect selection of bacterial mutants. J Bacteriol 1952; 63: 399-406. (PMCID: PMC169282) (PMID: 14927572)

10. Cavalli-Sforza LL, Lederberg J. Isolation of preadaptive mutants in bacteria by sib selection. Genetics 1956; 41: 367-381. (PMID: 17247634) (PMCID: PMC1209788)

11. Luria SE, Delbrück M. Mutations of bacteria from virus sensitivity to virus resistance. Genetics 1943; 28: 491-511. (PMID: 17247100) (PMCID: PMC1209226)

12. Newcombe HB. Origin of bacterial Variants. Nature 1949; 164: 150 151. (doi: 10.1038/164150a0)

13. Mittler JL, Lenski RE. New data on excisions of Mu from E. coli MCS2 cast doubt on directed mutation hypothesis. Nature 1990; 344: 173175. (PMID: 2407962) (doi: 10.1038/344173a0)

14. Mittler JE, Lenski RE. Experimental evidence for an alternative to directed mutation in the $\mathrm{Bgl}$ operon. Nature 1992; 356: 446-448. (PMID: 1557128) (doi: 10.1038/356446a0)

15. Lenski RE, Sniegowski PD. Directed mutations slip-sliding away? Curr Biol 1995; 5(2): 97-99. (PMID: 7743184)

16. Benson SA, Occi JL, Sampson BA. Mutations that alter the pore function of the OmpF porin of Escherichia coli K12. J Mol Biol 1988; 203: 961-970. (PMID: 2463371) (doi: 10.1016/0022-2836(88)90121-0)

17. Hall BG. Spontaneous point mutations that occur more often when advantageous than when neutral. Genetics 1990; 126: 5-16. (PMID: 2227388) (PMCID: PMC1204135)

18. Benson SA, DeCloux AM, Munro J. Mutant bias in nonlethal selections results from selective recovery of mutants. Genetics 1991; 129: 647658. (PMID: 1661253) (PMCID: PMC1204732)

19. Cairns J, Foster PL. Adaptive reversion of a frameshift mutation in Escherichia coli. Genetics 1991; 128: 695-701. (PMID: 1916241) (PMCID: PMC1204544) 
20. Stewart EJ, Madden R, Paul G, Taddei F. Aging and death in an organism that reproduces by morphologically symmetric division. PLOS Biol 2005; 3(2), e 45. (doi/10.1371/journal.pbio.0030045) (PMID: 15685293) (PMCID: PMC546039)

21. Luzzatto L, Apirion D, Schlessinger D. Mechanism of action of streptomycin in E. coli: interruption of the ribosome cycle at the initiation of protein synthesis. Proc Natl Acad Sci USA 1968; 60(3): 873880. (PMID: 4875806) (PMCID: PMC225133)

22. Rubin H. Central roles of Mg2+ and MgATP2- in the regulation of protein synthesis and cell proliferation: significance for neoplastic transformation. Adv Cancer Res 2005; 93: 1-58. (PMID: 15797443) (doi: 10.1016/S0065-230X(05)93001-7)

23. Hardie DG, Hawley SA. AMP-activated protein kinase: the energy charge hypothesis revisited. BioEssays 2001; 23(12): 1112-1119. (PMID: 11746230) (doi: 10.1002/bies.10009)

24. Pasternak K, Kocot J, Horecka A. Biochemistry of Magnesium. J Elementol 2010; 15(3): 601-616. (doi: 10.1016/j.indcrop.2012.04.059)

25. Takaya J, Iharada A, Okihana H, Kaneko K. Magnesium deficiency in pregnant rats alters methylation of specific cytosines in the hepatic hydroxysteroid dehydrogenase-2 promoter of the offspring. Epigenetics 2011; 6(5): 573-578. (doi: 10.4161/epi.6.5.15220/ PMID: 21406963)

26. Gomes MVM, Toffoli LV, Arruda DM et al. Age-Related Changes in the Global DNA Methylation Profile of Leukocytes Are Linked to Nutrition but Are Not Associated with the MTHFR C677T Genotype or to Functional Capacities. PLoS ONE 2012 7(12): e52570. (PMID: 24469919/ doi:10.1371/journal.pone.00525702)

27. Brown RA., Spina D, Page CP. Adenosine receptors and asthma. $\mathrm{Br} J$ Pharmacol 2008; 153(S1): S446-S456. (PMID: 18311158) (PMCID: PMC2268070) (doi: 10.1038/bjp.2008.22)

Authors:

Maurice Samuel Devaraj - Independent Researcher, Chennai, India. 